\title{
5G positioning and hybridization with GNSS observations
}

\author{
R.Crapart, Telespazio \\ R.Maymo-Camps, Telespazio \\ B.Vautherin, Thales Alenia Space \\ J.Saloranta, University of Oulu \\ Email: \\ romain.crapart@telespazio.com \\ roc.maymo-camps@telespazio.com \\ benoit.vautherin@thalesaleniaspace.com \\ jani.saloranta@oulu.fi
}

\section{BIOGRAPHIES}

Roc Maymo-Camps has had engineering masters by Escola Tècnica Superior d'Enginyeria Industrial de Barcelona (UPC) and ENAC (2007). He has been working at Telespazio for over 10 years in the navigation department.

Benoit Vautherin received his engineering degree from Ecole supérieure d'électricité, Metz, France, in 2015, and his MSc degree in space science and engineering from University College London, United Kingdom, in 2015. He joined Thales Alenia Space, Toulouse, France, in April 2015. Since then, he has been working as a navigation and data collect system engineer on many European and national $R \& D$ projects.

M.Sc Jani Saloranta is a PhD student at University of Oulu, Finland. He received his Master's degree in Mathematics (Group Theory) with minors from Statistics and Computer Sciences from University of Oulu in 2008. Since 2009 he has been a research scientist at Centre for Wireless Communications, University of Oulu, Finland. Since 2012 he is working towards his $\mathrm{PhD}$ degree from the title of Semantic Positioning in Department of Communications Engineering, University of Oulu, Finland.

Romain Crapart received his engineering degree from the Institut Supérieur de l'Aéronautique et de l'Espace ISAE (ENSICA course) in 2016. He joined Telespazio France in 2018 in the navigation department and is working as GNSS engineer for mobile and space applications.

\footnotetext{
ABSTRACT

The present paper depicts the tests and experimentations that have been carried out to show how GNSS and 5G can be used to improve positioning performance. In this frame, the design made during the $5 \mathrm{G}-$ Champion project has led to a solution combining both positioning systems in different environments to show in which cases one solution is better than the others and in which cases they can be complementary. The experimentations show the interest of
}

GNSS in open-sky and rural areas where 5G base stations will not be close to the user and will not be able to provide accurate positioning data. On the other extreme, in canyons and urban environments where buildings and multipath abundant can mask many satellites, the inclusion of 5G positioning data from stations that tend to be close to the user can improve the performance. At the end of this paper, a table summarizes the performance improvements, namely the accuracy and availability. Moreover, PPP solutions have been computed in order to see the benefits of PPP and 5G solutions combined.

\section{INTRODUCTION}

The paradigm of ubiquitous location information has risen a requirement for hybrid positioning methods, as a continuous data location cannot be provided by a single wireless system. Thus, such hybrid methods have interested the research community. Different joint techniques accounting different data sources and/or types have been proposed, e.g. coupling GNSS with vision sensors (Takasu \& Yasuda, 2008) or inertial sensors (Angrisano, 2010). Nevertheless, the upcoming fifth generation $(5 \mathrm{G})$ networks jointly with Global Navigation Satellite System (GNSS) are still to be investigated.

It is expected that $5 \mathrm{G}$ positioning will be able to provide more precise location and enhanced availability in all kinds of environments, especially urban canyons, where most GNSS signals are blocked and suffer from severe multipath conditions. The $5 \mathrm{G}$ observations can be added to both single and precise point positioning (PPP) GNSS solutions to improve positioning performance.

The work was carried out during the EU-Korean "5GCHAMPION" project (Mueck \& et.al., 2016). The paper is organized as follows.

- In Section 2, the objectives of the work are explained.

- In Section 3, the research methods and the presented objectives are discussed.

- In Section 4, the technologies and methods are explained from the positioning point of view for the 
proposed hybrid 5G-GNSS method and infrastructure.

- Then in Section 5, results based on simulations and real life measurements are presented,

- And finally, in Section 6 concluding remarks are given.

\section{OBJECTIVES}

This study has three main objectives:

- Assess the positioning performance of the mmWave technology.

- Assess the benefit of 5G positioning over known GNSS positioning methods.

- Assess the positioning performance of the hybridized mm-Wave/GNSS solution

\section{METHOD}

The fulfillment of the objectives is done in two phases.

First, each solution is described to provide a technical understanding and to assess preliminary performance of each technology.

Second, a testing phase is provided with definition of Key Performance Indicators (KPI) and test cases. Then the test cases are executed and post-processed to assess the performance of each solution in real life.

\section{TECHNOLOGY DEFINITION}

\section{1 mm-Wave Positioning}

The millimeter wave (mmWave) technology ${ }^{1}$ is considered as one of the key properties of the $5 \mathrm{G}$ communication networks (Saloranta \& Destino, 2017). The exact definition of mmWave states a frequency band of 30 - $300 \mathrm{GHz}$; a relaxed definition e.g. from $23-32 \mathrm{GHz}$ bands is considered in literature. This technology mainly brings the use of high data rate and the use of beamforming feature via large antenna arrays (Kutty \& Sen, 2016). Such feature is used for the angle of arrival (AOA) positioning method. Furthermore, the characteristic of the mmWave channel is sparsity, i.e. only a few dominant paths exist in the channel estimation process, and from where the spatialtemporal domain information can be exploited in the estimation process. (Saloranta \& Destino, 2016). To provide an effective solution utilizing a real hardware, we exploit the angular domain information only.

The beamforming algorithm is done in two phases depicted in Figure 1 and Figure 2. The first phase consists in

1. Dividing the 60deg aperture into 8 beams,

2. Selecting the max SNR beam and its neighbors, The second phase consists in

1. Dividing the 3 previously selected beams in 8 ,

2. Selecting the maximum SNR beam.

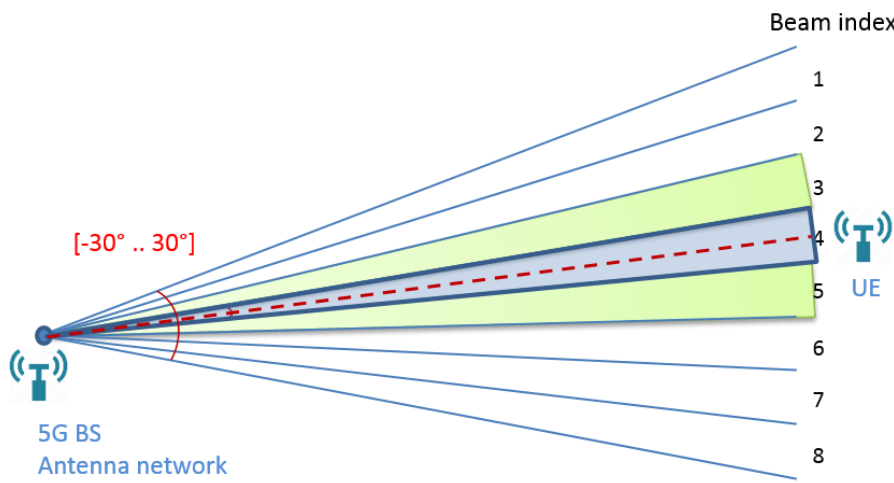

Beam cover $=$ Beam center $+\left[-3.75^{\circ} . .3 .75^{\circ}\right]$

Figure 1: $1^{\text {st }}$ phase of beam selection

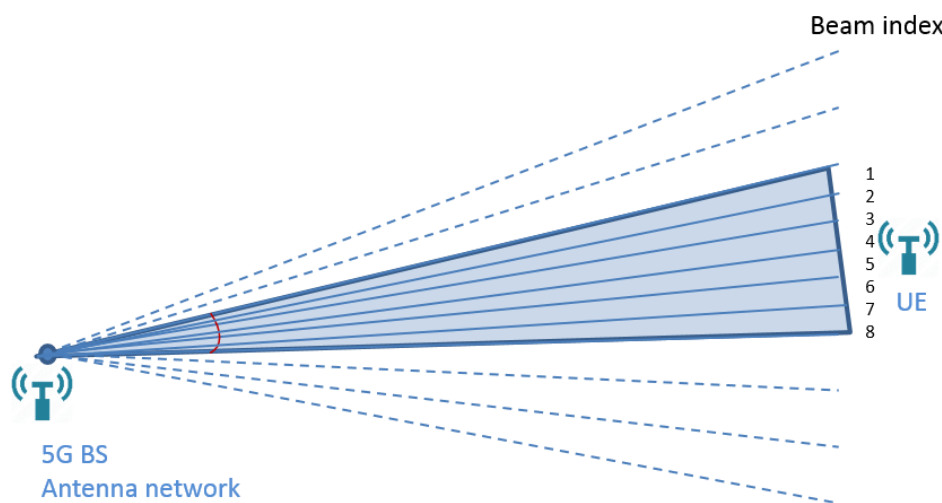

Figure 2: $2^{\text {nd }}$ phase of beam selection

At the end of the beam selection process the obtained angular resolution of the selected beam is $2.8 \mathrm{deg}$. As a consequence, the angular error model was chosen to be a uniform distribution as depicted in Figure 3.

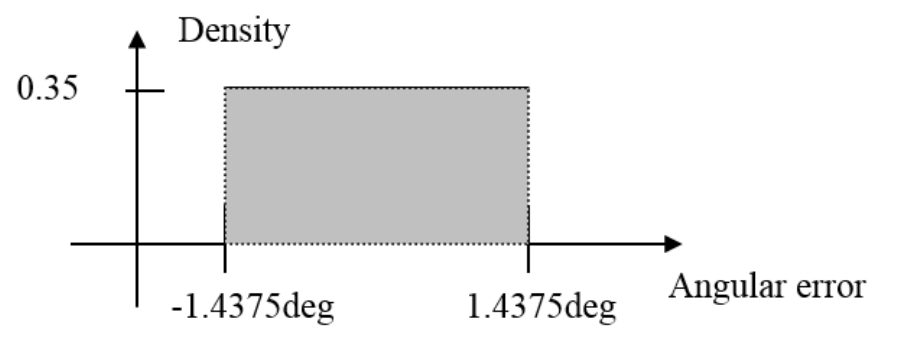

Figure 3: Angular error model

\subsection{Single Positioning and Hybridization}

$5 \mathrm{G}$ observations are azimuth and elevation angles as defined in Figure 4.

\footnotetext{
${ }^{1}$ A radio frequency band of $30-300 \mathrm{GHz}$ via exact definition. Although relaxed definition are e.g. from $23-32 \mathrm{GHz}$ bands [P1].

International Technical Symposium on Navigation and Timing (ITSNT) 2018

13-16 Nov 2018

ENAC, Toulouse, France
} 


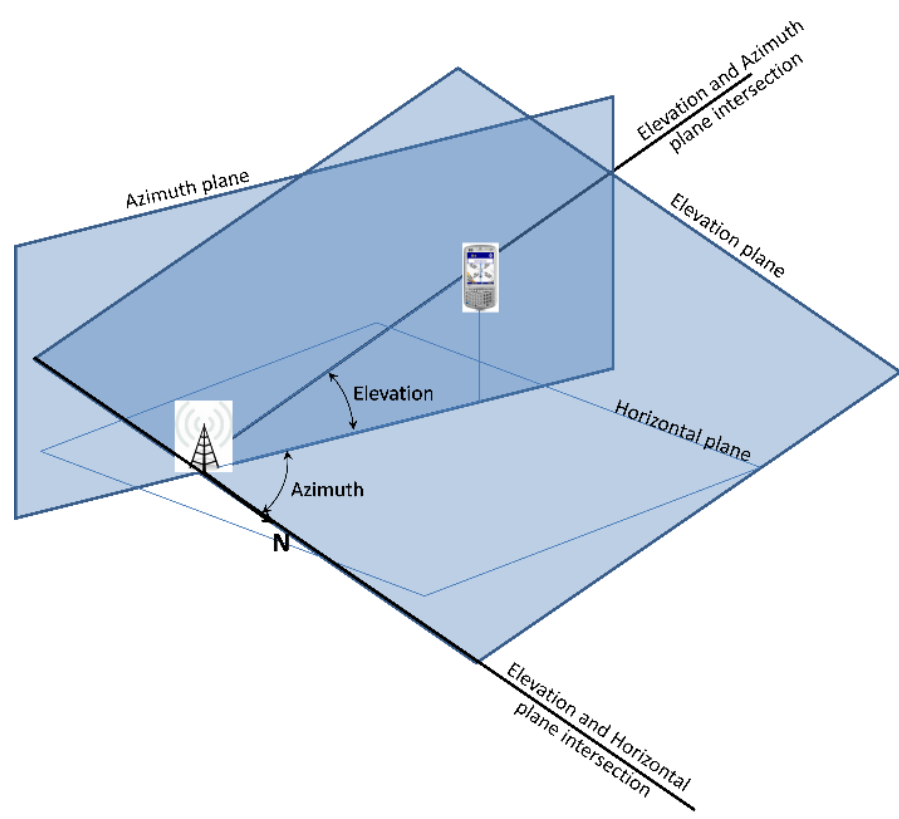

Figure 4: Azimuth and Elevation planes

Explanation of single point positioning (SPP) is not the objective of this study but the main mathematical implementation resides in the following equations. $5 \mathrm{G}$ observations are in angular domain (azimuth and elevation that can be represented as a line from tower to cell) and are added to the observation model as 2 planes representing the line. This system is given as

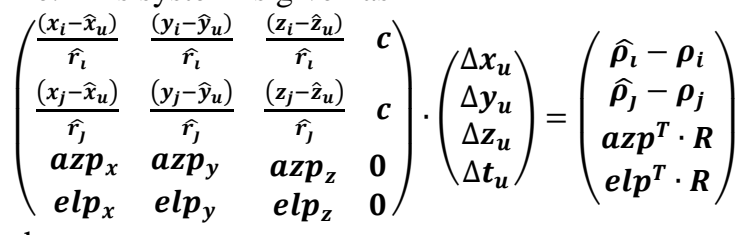

where

- $\left(\begin{array}{llll}\Delta x_{u} & \Delta y_{u} & \Delta z_{u} & \Delta t_{u}\end{array}\right)$ : State vector residuals

- $\left(\begin{array}{lll}\hat{x}_{u} & \hat{y}_{u} & \hat{z}_{u}\end{array}\right)$ : Estimated user position

- $\left(\begin{array}{lll}x_{i} & y_{i} & z_{i}\end{array}\right)$ : Estimated position of satellite $i$

- $\widehat{r}_{l}$ : Estimated range between receiver and satellite $i$

- $\widehat{\rho}_{l}$ : Estimated pseudo-range between receiver and satellite $i$

- $\quad \rho_{i}$ : GNSS observation of pseudo-range $i$

- $\quad c$ : The constant of the speed of light

- $\quad R$ : Range between estimated user position and base station (BS) expressed in ECEF.

- $\quad a z p=\left(\begin{array}{lll}a z p_{x} & a z p_{y} & a z p_{z}\end{array}\right):$ Azimuth plane vector expressed in ECEF coordinates. The vector is orthogonal to the azimuth plane.

- $\quad e l p=\left(\begin{array}{lll}e l p_{x} & e l p_{y} & e l p_{z}\end{array}\right):$ Elevation plane vector expressed in ECEF coordinates. The vector is orthogonal to the elevation plane.

The associated covariance matrix $\Sigma$ of observations is as

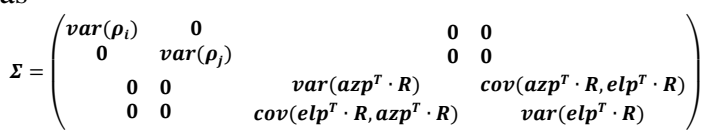

The variance of GNSS observation is based on the User Range Equivalent Error (UERE), which is well documented. The variance of $5 \mathrm{G}$ observation is quadratic with the distance of the estimated position from the station which comes from the angular nature of the observation as presented in Equation 2.

\subsection{Precise Point Positioning and Hybridization}

Based on undifferenced and uncombined code and phase measurements, Precise Point Positioning (PPP) techniques can reach decimeter level accuracy in kinematic mode and centimeter level or better in static mode thanks to the precise orbit, clock and error models. This paper presents the results of PPP-WIZARD (With Integer and Zero-Difference Ambiguity Resolution) software hybridized with 5G observations. This software uses recent techniques such as ambiguity resolution, fast convergence, gap bridging, etc. and presents the possibility of single frequency PPP (Laurichesse \& Privat, 2015). These techniques improve the accuracy and the convergence time of the solution.

The GNSS/5G hybridization consisted mainly in adding $5 \mathrm{G}$ observations in the measurement vector of the PPP Extended Kalman Filter, and completing the covariance matrices according to the equations (1) and (2). We used as hypothesis the fact that $5 \mathrm{G} \mathrm{BS}$ and the UE are both in the azimuth plane:

$$
\begin{gathered}
\operatorname{azp}_{x} x_{u}+\operatorname{azp}_{y} y_{u}+\operatorname{azp}_{z} z_{u}=\operatorname{azp}_{x} X_{S}+\operatorname{azp}_{y} Y_{S}+\operatorname{azp}_{z} Z_{S} \\
e l p_{x} x_{u}+e l p_{y} y_{u}+e l p_{z} z_{u}=e l p_{x} X_{S}+e l p_{y} Y_{S}+e l p_{z} Z_{S}
\end{gathered}
$$

with $(X s, Y s, Z s)$ and $\left(x_{u}, y_{u}, z_{u}\right)$ respectively the BS and the user coordinates in ECEF.

The state vector (estimated parameters) is as:

$$
\begin{aligned}
\Delta \boldsymbol{X}= & {\left[\boldsymbol{P}, \boldsymbol{C l} \boldsymbol{k}_{1, . . n}, \boldsymbol{B}_{f i_{1}, . . n}, \Delta \boldsymbol{T r}, \boldsymbol{s} \boldsymbol{I}, \boldsymbol{N}\right] } \\
& -\quad P: \text { position vector }\left(x_{u}, y_{u}, z_{u}\right) \\
& -\quad C l k_{1, . . n}: \text { receiver clock for each constellation } \\
& -\quad B_{f i_{1, \ldots n}}: \text { estimated biases for each } \\
& \quad \text { constellation and frequency } \\
& -\quad \Delta T r: \text { zenithal tropospheric delay } \\
& -\quad s I: \text { slant ionospheric delay } \\
& -\quad N: \text { phases ambiguities } \\
& -\quad n: \text { number of constellations }
\end{aligned}
$$




\section{RESULTS}

\subsection{Test Architecture}

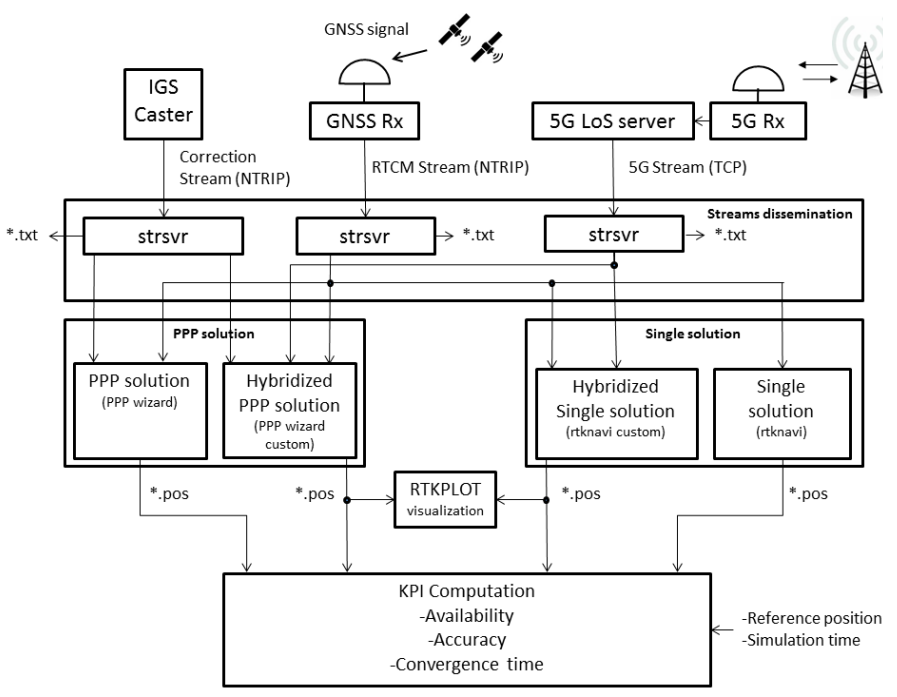

Figure 5: Test Bed architecture

The PPP corrections are retrieved from an IGS caster, and the GNSS observations are recorded from a mass market receiver (Samsung Galaxy S8 or ublox M8T). They are provided as RTCM streams through an NTRIP connection. $5 \mathrm{G}$ observations are provided through a TCP stream. To obtain results as close as possible to realistic beamforming antennas, only azimuth angles are included as $5 \mathrm{G}$ observation. The elevation angle is excluded and the hybridization algorithms receives one extra observation for each BS (instead of two observations).

The hybridization with Precise Point Positioning is computed with the customized software PPPwizard, while the hybridization with single point positioning (SPP) solution is computed with the customized rtknavi software from the open source suite RTKLIB.

Each solution is provided under the same file format and post-processed with a Matlab code.

\subsection{KPI}

The defined KPI are the availability, the accuracy and the convergence time (to obtain an accuracy better than 1 meter) of the solution.

\subsection{Test Cases}

The GNSS test environment are visibility conditions and are chosen to be clear-sky, urban and canyon environments. GPS, GLONASS and GALILEO constellations are used in the L1 band in order to be as close as possible to current mass market receivers.

The $5 \mathrm{G}$ conditions are the relative position of the $5 \mathrm{G}$ base stations (BS) with respect to the User Equipment (UE). The following BS locations are used:

- N20: North 20 meters (azimuth angle equals 180 deg from the $5 \mathrm{G} B \mathrm{BS}$ )

- N20 E20: North 20 meters and East 20 meters (two 5G BS: 180deg and 270deg)

- N20 SE20: North 20 meters and South-East 20 meters (two 5G BS: 180deg and 315deg)

Same locations were also tested using BS at $50 \mathrm{~m}$ from the UE.

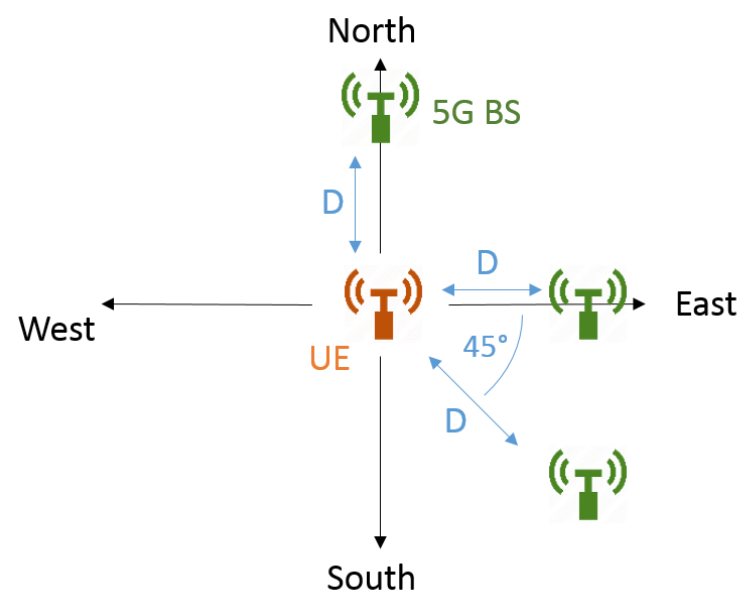

Figure 6: $5 G \mathrm{BS}$ locations with $D=20 \mathrm{~m} \& \mathrm{D}=50 \mathrm{~m}$

\subsection{Compared Performance}

For computing the positioning accuracy a georeferenced position was use for the open-sly environment and a precisely computed position for the urban and canyon environment.

\subsubsection{Clear Sky}

\subsubsection{N50}

The user is placed at $50 \mathrm{~m}$ from the $5 \mathrm{G}$ BS placed at North.

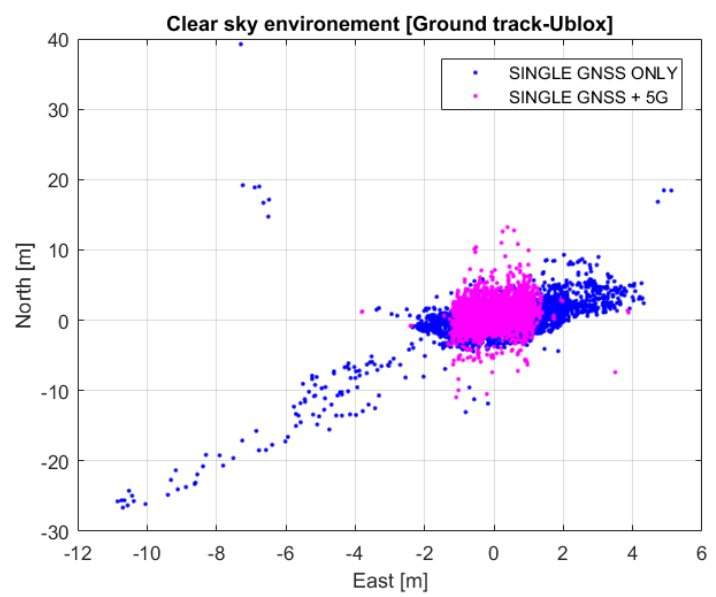

Figure 7: Ground track (SPP) with 1 BS at 50m North The ground track visualization highlights the benefits of the extra observation for the perpendicular direction to the BS. In this case the dilution of the results became closer to the UE along the East-West direction. This observation is confirmed by the true error applied on this direction and provided on the figure hereafter. 


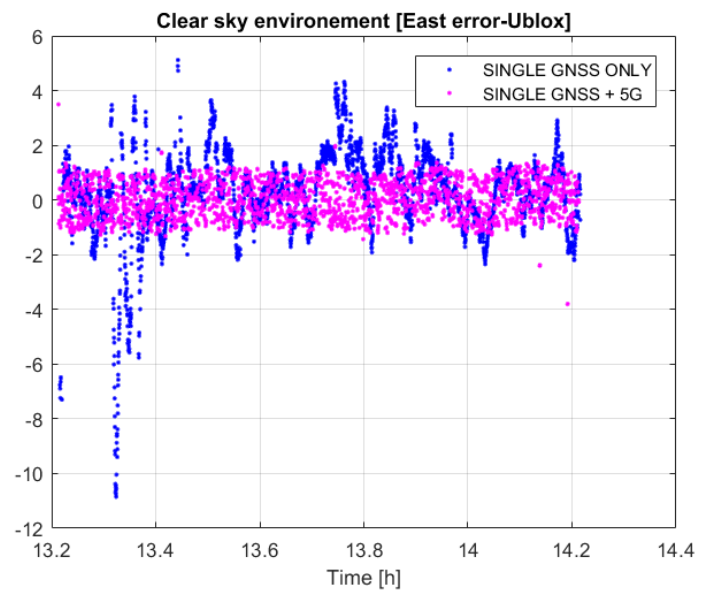

Figure 8: East errors (SPP) with 1 BS at 50m North As expected from the ground track figure, the BS located at the North of the UE reduces the error on the East-West direction.

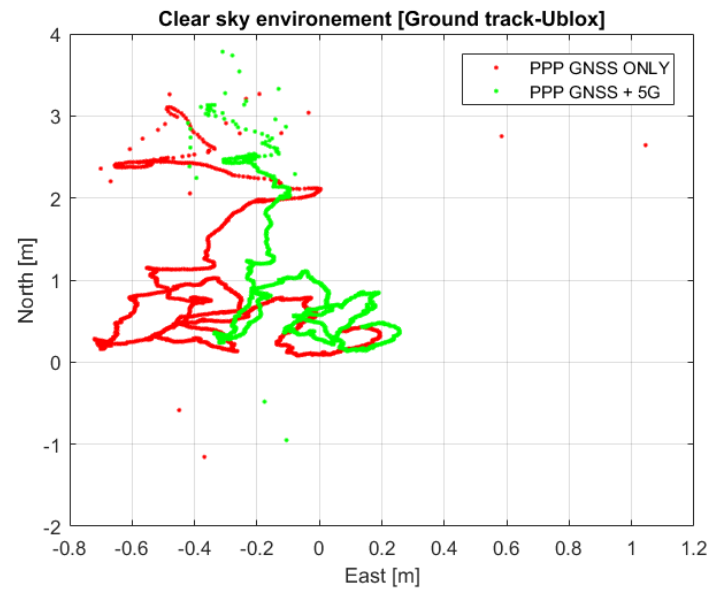

Figure 9: Ground track (PPP) with 1 BS at 50m North

The same behavior can be observed on the ground track of the PPP solution as for the SPP. Nevertheless the North error dilution (the major contribution of the error in this case) is not affected by the extra observation.

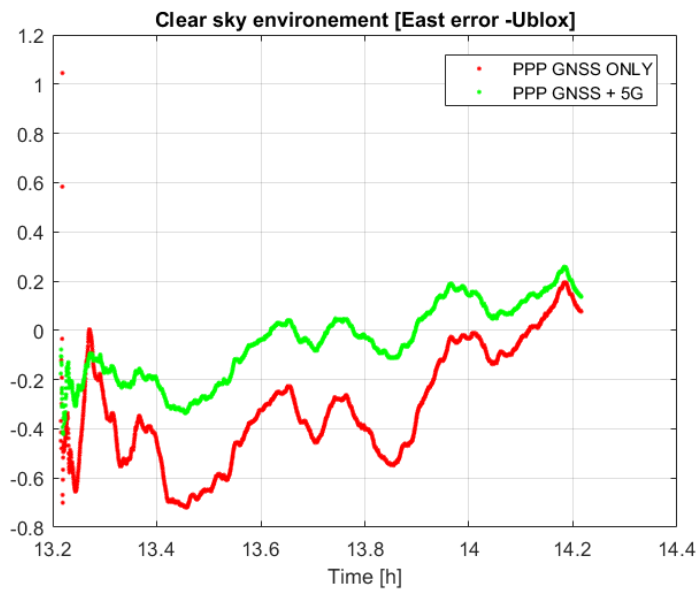

Figure 10: East errors (PPP) with 1 BS at 50m North As foreseen from the ground track visualization, a slight improvement of the error can be observed on the East direction.

\subsubsection{N20 E20}

The user is placed at $20 \mathrm{~m}$ from two $5 \mathrm{G}$ BS located at East and North.

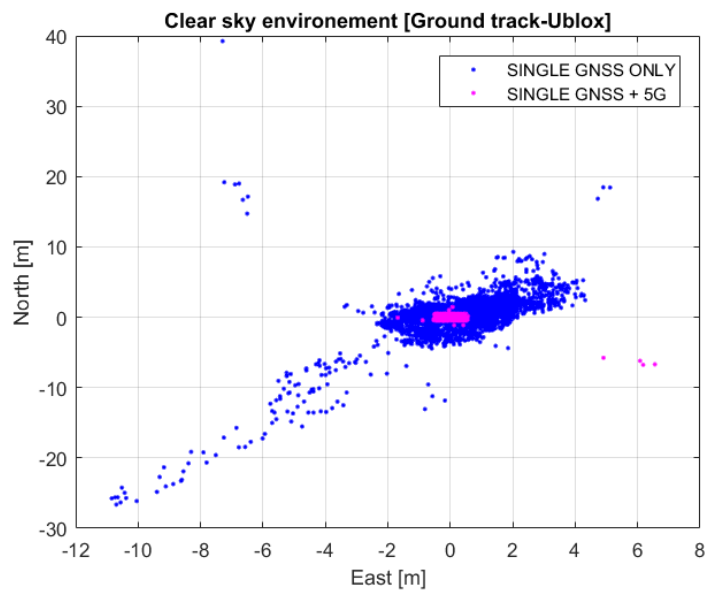

Figure 11: Ground track (SPP) with 2 BS at 20m North \& $20 m$ East

When 2 BS are visible from the UE, 2 extra observations are available. Using BS spaced from 90deg allows the UE to benefit from optimal combination of observations. The BS located at the North (resp. East) provides azimuth angle that narrows the solution on the East-West (resp. NorthSouth) direction.

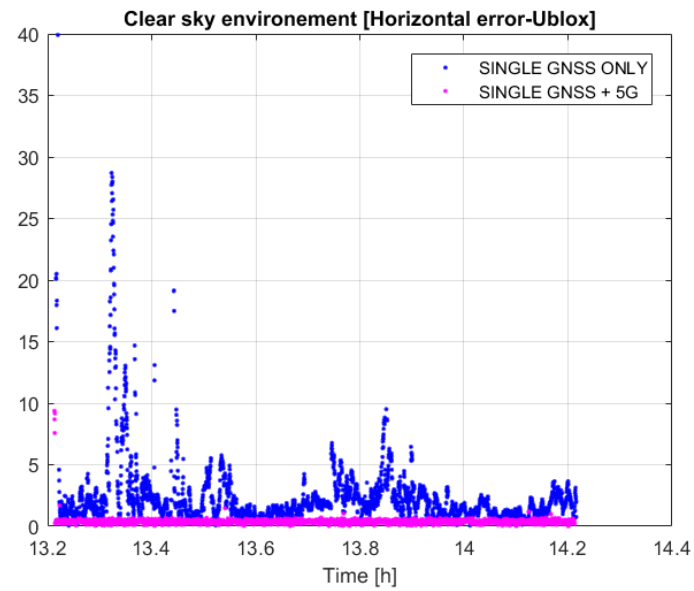

Figure 12: Horizontal errors (SPP) with 2 BS at 20m North \& $20 m$ East

Indeed the true horizontal error is greatly improved when using BSs $20 \mathrm{~m}$ away from the UE.

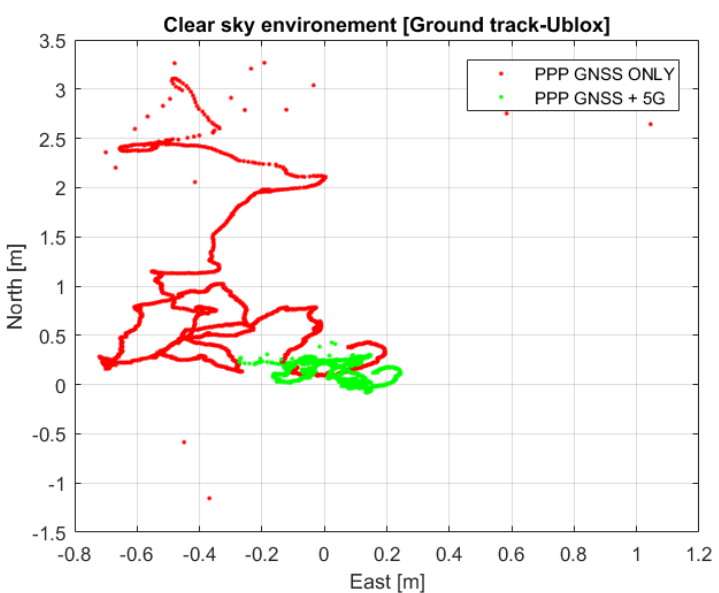

Figure 13: Ground track (PPP) with 2 BS at 20m North \& $20 m$ East

The same behavior as for the SPP can be observed on the ground track of the PPP solution resulting on an improved dilution on both North-South and East-West direction.

International Technical Symposium on Navigation and Timing (ITSNT) 2018

13-16 Nov 2018

ENAC, Toulouse, France 


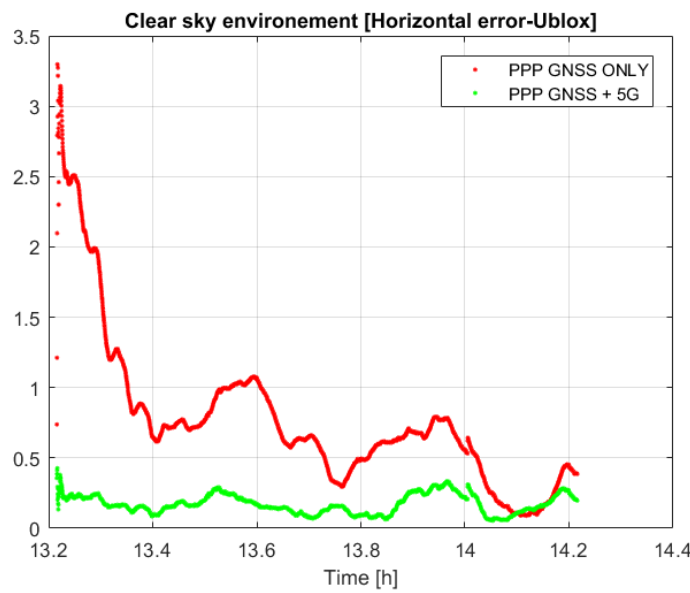

Figure 14: Horizontal errors (PPP) with 2 BS at 20m North \& 20m East

As expected from the ground track figure, a great improvement of the horizontal error can be observed.

\subsubsection{Urban}

\subsubsection{N20 SE20}

The user is placed at $20 \mathrm{~m}$ from two $5 \mathrm{G}$ BS placed at South-East and North.

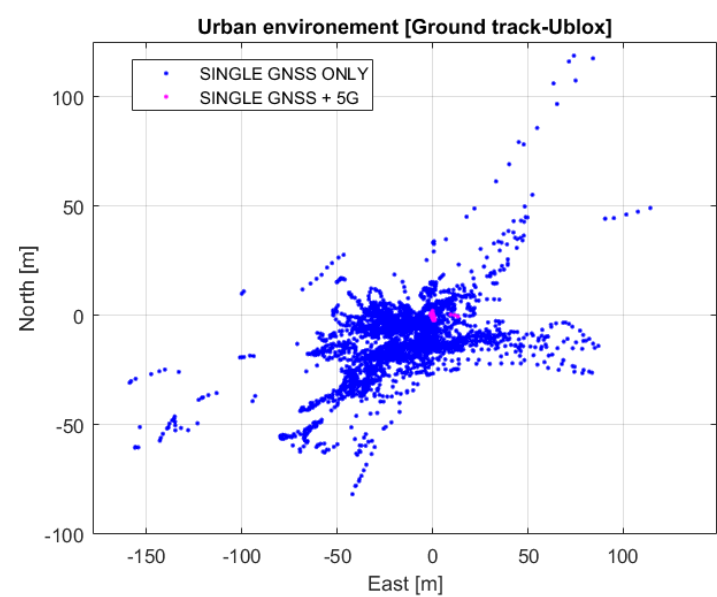

Figure 15: Ground track (SPP) with 2 BS at 20m North \& 20m South-East

Under urban environment the dilution of the SPP horizontal error is degraded w.r.t. the open sky conditions. However the hybridized SPP dilution stays close to the UE even with low quality GNSS signals.

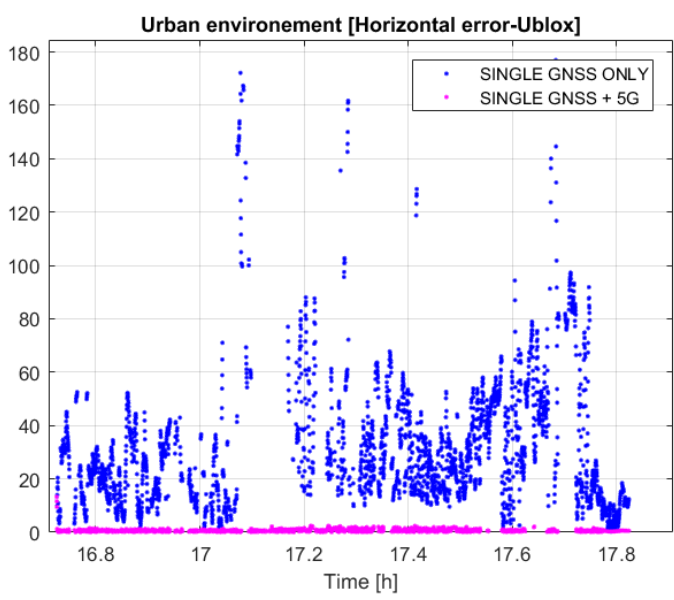

Figure 16: Horizontal errors (SPP) with 2 BS at 20m North \& 20m South-East

As expected from the ground track figure, a great improvement of the horizontal error can be observed.

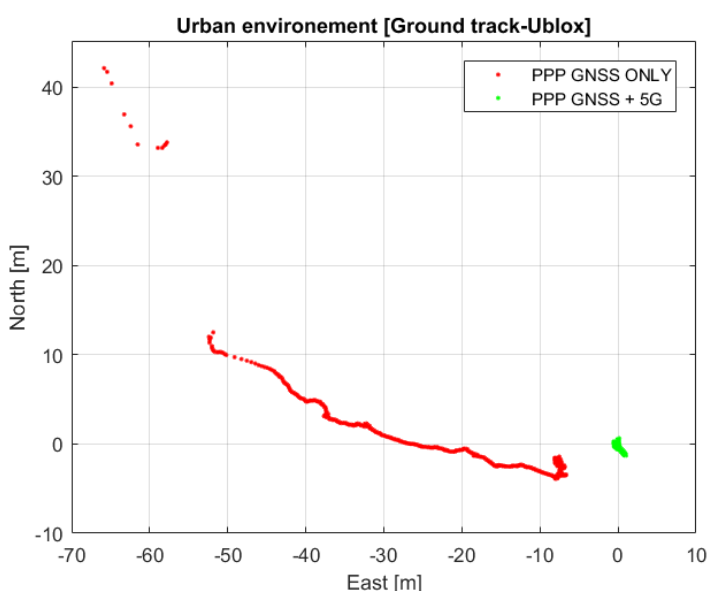

Figure 17: Ground track (PPP) with 2 BS at 20m North \& 20m South-East

Under urban environment, the PPP solution does not provide sub-meter accuracy while the addition of 2 BS allows the precise positioning with sub-meter accuracy at $99^{\text {th }}$ percentile.

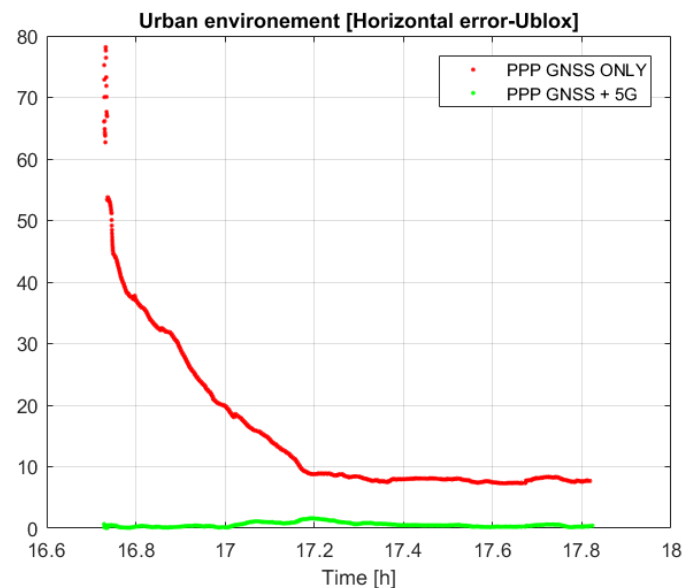

Figure 18: Horizontal errors (PPP) with 2 BS at 20m North \& 20m South-East

The convergence process is greatly shortened by the use of 2 extra observations as the first fix is already close to the $\mathrm{UE}$ (under 5m).

\subsection{Summary of the results}

The results of this study are summarized in the Table 1 . The canyon scenario was used to observe the limits of the solution proposed by reusing the input data from the urban scenario adding a satellite mask of $45^{\circ}$ and using GPS only which leads to solutions with 3 satellites only.

\begin{tabular}{|c|c|c|c|c|c|c|c|c|c|}
\hline \multirow{2}{*}{ Test Case } & \multirow{2}{*}{ Conf. } & \multirow{2}{*}{ Method } & \multirow{2}{*}{$\begin{array}{c}\text { Availability } \\
{[\%]}\end{array}$} & \multicolumn{5}{|c|}{ Accuracy $[\mathrm{m}]$} & \multirow{2}{*}{$\begin{array}{r}\text { Convergenc } \\
\text { time [mn] }\end{array}$} \\
\hline & & & & Mean & Std & 70th pretl & 95th pretl & 99th prctl & \\
\hline \multirow{8}{*}{ Clear sky } & \multirow{2}{*}{ No 5G } & PPP & 99.917 & 0.492 & 0.224 & 0.634 & 0.790 & 0.964 & 23.53 \\
\hline & & Single & 98.89 & 1.845 & 1.279 & 2.147 & 4.244 & 6.448 & $\mathrm{NA}$ \\
\hline & \multirow{2}{*}{ N50 } & $\begin{array}{l}\text { PPP 5G } \\
\end{array}$ & 99.917 & 0.483 & 0.213 & 0.602 & 0.860 & 0.962 & 23.86 \\
\hline & & Single. 5G & 99.972 & 1.635 & 1.165 & 1.873 & 4.063 & 5.754 & $\mathrm{NA}$ \\
\hline & \multirow{2}{*}{ N20 E20 } & PPP 5G & 99.917 & 0.172 & 0.068 & 0.216 & 0.287 & 0.320 & 0 \\
\hline & & Single. 5G & 99.74 & 0.381 & 0.150 & 0.467 & 0.595 & 0.663 & $\mathrm{NA}$ \\
\hline & \multirow{2}{*}{ N20 SE20 } & PPP 5G & 99.917 & 0.265 & 0.131 & 0.331 & 0.466 & 0.623 & 0 \\
\hline & & Single. 5G & 99.722 & 0.518 & 0.273 & 0.622 & 1.008 & 1.160 & $\mathrm{NA}$ \\
\hline \multirow{3}{*}{ Urban } & \multirow{2}{*}{ No 5G } & PPP & 91.722 & 15.174 & 11.509 & 16.068 & 37.975 & 53.170 & No Conv. \\
\hline & & Single & 72.645 & 41.789 & 101.768 & 40.389 & 84.826 & 175.059 & $\mathrm{NA}$ \\
\hline & $\mathrm{N} 20$ & PPP 5G & 91.722 & 2.012 & 2.070 & 2.269 & 3.002 & 4.023 & No Conv. \\
\hline
\end{tabular}

International Technical Symposium on Navigation and Timing (ITSNT) 2018

13-16 Nov 2018

ENAC, Toulouse, France 


\begin{tabular}{|c|c|c|c|c|c|c|c|c|c|}
\hline & & Single. 5G & 77.645 & 14.504 & 30.005 & 14.872 & 31.662 & 114.191 & $\mathrm{NA}$ \\
\hline & \multirow{2}{*}{ N20 E20 } & PPP 5G & 91.722 & 0.492 & 0.202 & 0.597 & 0.836 & 0.923 & 28.217 \\
\hline & & Single. 5G & 72.396 & 0.430 & 0.198 & 0.516 & 0.745 & 0.918 & NA \\
\hline & \multirow{2}{*}{ N20 SE20 } & PPP 5G & 91.764 & 0.479 & 0.208 & 0.572 & 0.858 & 0.949 & 31.8 \\
\hline & & Single. 5G & 72.757 & 0.725 & 0.448 & 0.904 & 1.608 & 1.901 & $\mathrm{NA}$ \\
\hline \multirow{8}{*}{ Canyon } & \multirow{2}{*}{ NO 5G } & PPP & 0 & No fix & No fix & No fix & No fix & No fix & NA \\
\hline & & Single & 0 & No fix & No fix & No fix & No fix & No fix & NA \\
\hline & \multirow[b]{2}{*}{$\mathrm{N} 20$} & PPP 5G & 49.669 & 405.91 & 428.39 & 397.17 & 1402.5 & 1421.5 & $\mathrm{NA}$ \\
\hline & & Single. 5G & 39.458 & 2256.062 & $\begin{array}{c}14277.03 \\
3 \\
\end{array}$ & 1310.511 & 4880.827 & \begin{tabular}{|c}
28832.35 \\
3 \\
\end{tabular} & $\mathrm{NA}$ \\
\hline & \multirow{2}{*}{ N20 E20 } & PPP 5G & 61.556 & 341.22 & 277.27 & 384.71 & 835.27 & 1277.5 & $\mathrm{NA}$ \\
\hline & & Single. 5G & 66.105 & 0.4006 & 0.1848 & 0.4787 & 0.6449 & 0.7272 & NA \\
\hline & \multirow{2}{*}{ N20 SE20 } & PPP 5G & 61.556 & 328.55 & 220.86 & 386.21 & 715.44 & 1020.60 & $\mathrm{NA}$ \\
\hline & & Single. 5G & 66.157 & 0.517 & 0.280 & 0.605 & 1.029 & 1.197 & $\mathrm{NA}$ \\
\hline
\end{tabular}

Table 1: Summary of results

For the canyon tests, only the solutions with $5 \mathrm{G}$ are possible. The test with a single $5 \mathrm{G}$ station shows extremely bad results. When checking the geometry of the solution, it appears that the 3 satellites used in the solution are aligned East-West. These leads to a geometric solution like a line North-South which is parallel to the plain equation provided by the $5 \mathrm{G}$ station. With such geometric conditions the user position in only observable on the EastWest direction which leads to a bad positioning accuracy on the North-South direction.

\section{CONCLUSIONS}

The addition of a $5 \mathrm{G}$ antenna clearly improves the positioning accuracy in the direction perpendicular to the antenna by providing extra observations to the PVT algorithms. When the maturity of the beamforming antennas will allow the use of elevation angle the positioning accuracy is expected to be even better than the ones obtained during this project.

Sub-metric accuracy is achievable even in an urban environment with the use of two perpendicular 5G antennas $20 \mathrm{~m}$ apart. Nevertheless the reader should know that the use of one BS in a perpendicular direction of a street under canyon environment could result in bad solution accuracy.

The outcome of these experimentations have shown the added value of the $5 \mathrm{G}$ when it comes to positioning performances (both for SPP and PPP). As observed in the last table, the $5 \mathrm{G}$ is especially important in the urban environments where GNSS signals are less available and the base stations are close enough. On the other hand, the interest of using $5 \mathrm{G}$ diminishes with the distance to the base station and one can imagine that in a rural environment, $5 \mathrm{G}$ positioning will not improve GNSS.

\section{ACKNOWLEDGMENTS}

The research leading to these results has received funding from the European Union H2020 5GPPP under grant n. 723247 and supported by the Institute for Information \& communications Technology Promotion (IITP) grant funded by the Korea government (MSIP) (No.B0115-16-0001, 5GCHAMPION).

\section{REFERENCES}

5GChampion. (2018). Deliverable D6.3 - Integration and system testing phase of satellite scenario.
(2018). D6.3 Integration and system testing phase of satellite scenario.

Kutty, S., \& Sen, D. (2016). Beamforming for Millimeter Wave Communications: An Inclusive Survey. IEEE Communications Surveys \& Tutorials, 18(2), 949-973.

Mueck, M., \& et.al. (2016). 5G CHAMPION - Rolling out 5G in 2018. Proc. IEEE Global Commun. Conf. Workshops, (pp. 1-6).

Saloranta, J., \& Destino, G. (2016). On the Utilization of MIMO-OFDM Channel Sparsity for Accurate Positioning. 24th European Signal Processing Conference (EUSIPCO), (pp. 748-752).

Saloranta, J., \& Destino, G. (2017). Reconfiguration of 5G radio interface for positioning and communication. 25th European Signal Processing Conference (EUSIPCO). 